\title{
Relativistic Solar Protons on 1989 October 22: Injection and Transport along Both Legs of a Closed Interplanetary Magnetic Loop
}

\author{
David Ruffolo $^{a}$, Paisan Tooprakai ${ }^{b}$, Manit Rujiwarodom ${ }^{b}$, Thiranee Khumlumlert ${ }^{c}$, \\ Maneenate Wechakama ${ }^{d}$, John W. Bieber ${ }^{e}$, Paul Evenson $^{e}$ and Roger Pyle ${ }^{e}$ \\ (a) Dept. of Physics, Faculty of Science, Mahidol Univ., Bangkok 10400, Thailand \\ (b) Dept. of Physics, Faculty of Science, Chulalongkorn Univ., Bangkok 10330, Thailand \\ (c) Dept. of Physics, Faculty of Science, Naresuan Univ., Phitsanulok 65000, Thailand \\ (d) Dept. of Physics, Faculty of Science, Kasetsart Univ., Bangkok 10900, Thailand \\ (e) Bartol Research Inst., Univ. Delaware, Newark, DE 19716, USA \\ Presenter: John W. Bieber (john@bartol.udel.edu), usa-bieber-J-abs2-sh21-poster
}

Worldwide neutron monitor observations of relativistic solar protons on 1989 October 22 have proven puzzling, with an initial spike at some stations followed by a hump with bidirectional flows and a very slow decay. We analyze data from polar monitors, which measure the directional distribution of solar energetic particles (mainly protons) at rigidities of $\sim 1-3 \mathrm{GV}$. The inferred density and anisotropy are simultaneously fit by simulating the particle transport for various magnetic field configurations and determining the best-fit injection function near the Sun. The data are not well fit for an Archimedean spiral field, a magnetic bottleneck beyond Earth, or particle injection along one leg of a closed magnetic loop. A model with simultaneous injection along both legs of a closed loop provides the best explanation. Refined fits indicate a very low spectral index of turbulence, $q<1$, and hence an unusually low correlation length of magnetic fluctuations in the loop, a loop length of $4.7 \pm 0.3 \mathrm{AU}$, and escape from the loop on a time scale of 3 hours.

\section{Introduction}

The observations of relativistic solar protons by ground-based neutron monitors on 1989 October 22 have defied conventional explanations and proven mysterious for 15 years. Observations of relativistic solar protons during a ground-level enhancement (GLE) typically begin with a rapid, anisotropic onset, with most particles moving anti-Sunward along the interplanetary magnetic field. Because of pitch-angle scattering, which eventually leads to spatial diffusion, the distribution becomes more isotropic with time, and gradually decreases (or "decays") as particles diffuse out of the inner heliosphere. However, the GLE of 1989 October 22 had an extraordinary spike at onset, which was highly anisotropic. The event was also unusual in exhibiting a second peak, which we call the "hump," an hour after the initial spike, followed by a very slow decay.

While the spike can be interpreted [1] as the "coherent pulse" predicted by focused transport theory [2] for conditions of weak scattering (i.e., a long scattering mean free path), the hump and slow decay have proven more difficult to understand. Ref. [3] considers propagation along a standard Archimedean spiral field and two separate injections of particles at the Sun. However, a detailed analysis reveals bidirectional fluxes in the hump [4], which along with the slow decay is not explained by the model of [3]. A different line of reasoning was presented by $[4,5]$, who explained the spike and hump in terms of a "disturbed plasma region" beyond Earth that scattered particles back. The outward component of the bidirectional flow is attributed to extended solar injection. Actually, a more efficient type of backscattering is mirroring by a magnetic bottleneck beyond Earth; [6] show evidence for this process during the GLE of Bastille Day 2000. In the present work, we consider various magnetic configurations that might explain this mysterious data set. 


\section{Methods}

To analyze the distribution of relativistic solar protons, the total count rate from each polar neutron monitor was corrected for atmospheric pressure variations using separate absorption lengths for Galactic and solar particles. We thereby obtain the percentage increase of each station's counting rate over the GCR background. The percentage increase at the asymptotic direction for each station is fitted to a second-order Legendre polynomial. The first two Legendre coefficients of the directional distribution (density and weighted anisotropy) are fitted as a function of time. During the time of the initial spike with high anisotropy, 17:55-18:10 UT, only two neutron monitor stations, McMurdo and South Pole, registered a noticeable increase in count rate. For this time period, we therefore determine lower limit values for the density and weighted anisotropy, which were enforced when fitting the time profiles.

To model the density and weighted anisotropy profiles, We first solve a transport equation that takes into account pitch angle scattering and adiabatic focusing [7] using the numerical method of [8]. In order to represent the momentum distribution of the primary relativistic protons to which the neutron monitors are responding, we perform simulations for momentum values corresponding to the 5 th, 15 th, ..., 95 th percentile rigidities for the spectral index of 5.9. Results for these 10 momentum values are averaged to determine the density, weighted anisotropy, and curvature expected near the Earth. For the pitch-angle scattering coefficient, we use the standard parameterization $A|\mu|^{q-1}\left(1-\mu^{2}\right)$ and relate $A$ to the scattering mean free path $\lambda_{\|}$as described by [7]. We initially use a value of $q=1.5$, which was found to provide a good fit to the GLE of 2000 July 14 [6].

In this analysis, we consider three types of magnetic field configurations as shown in Figure 1. For a given magnetic field configuration and transport model parameters, we simultaneously fit the time profiles of density and weighted anisotropy to determine the optimal piecewise linear injection function. The injection function is defined as the rate at which particles are injected at a solar footpoint of the Sun-Earth magnetic field line as a function of time. We evaluate the goodness of fit with a $\chi^{2}$ statistic and thereby determine the optimal transport parameters and magnetic field configuration. [6] compared results for the GLE of 2000 July 14 from this procedure with independent simulation and fitting techniques, and found close agreement.

\section{Results and Conclusions}

After fitting the data and optimizing the injection profile at the Sun by the procedure of [9], we find that the magnetic configurations of a standard Archimedean spiral, a bottleneck beyond Earth, and a closed loop with injection along one leg all fail to explain the data (see Figure 2). On the other hand, a model with simultaneous injection along the two legs of the loop is able to fit the data.

In addition to the fits described above, we performed refined fits in the context of injection along both legs of the loop. We simultaneously fit the first three Legendre coefficients of the directional distribution as a function of time up to 24:00 UT on 1989 October 22. We explored the influence of $q$, the power-law index of the turbulence power spectrum, which affects the form of the scattering coefficient.

The following are the overall conclusions of our analysis of data from 9 polar neutron monitors for the unusual ground level enhancement of relativistic solar protons on 1989 October 22:

1. The key features of the density and anisotropy profiles are not well explained by magnetic field configurations of an Archimedean spiral, a bottleneck beyond Earth, or a loop with injection along one leg. They are well explained by injection along both legs of a closed interplanetary magnetic loop that included the Earth. 

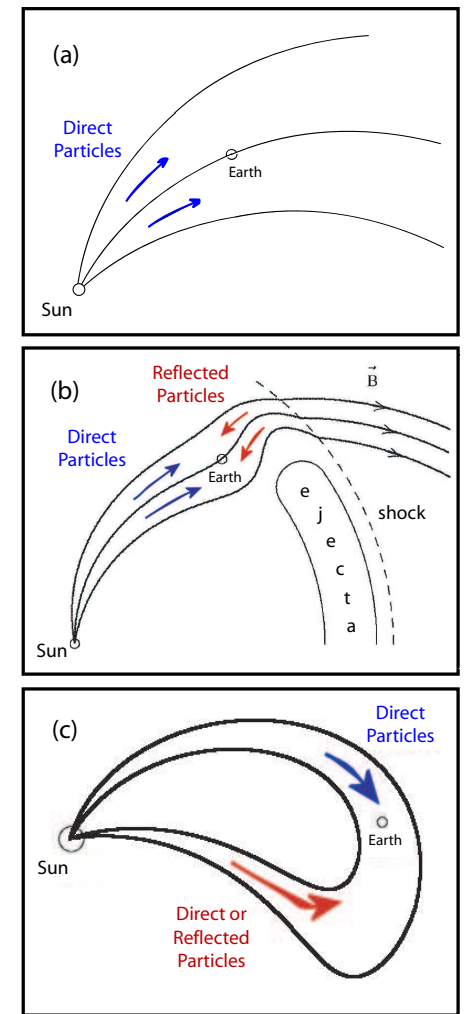
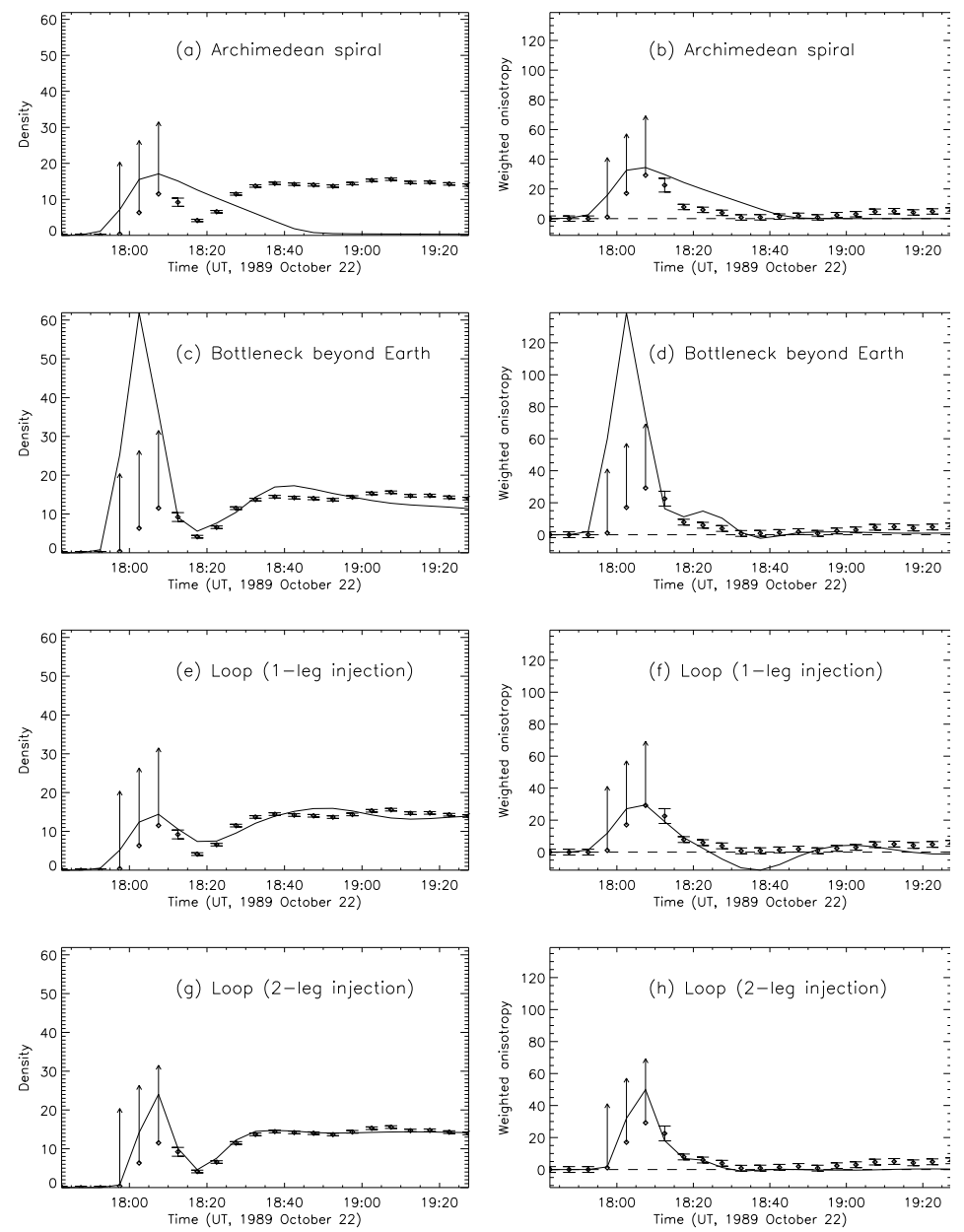

Figure 1. (left side) The three magnetic field configurations considered in this work: (a) standard Archimedean spiral, (b) magnetic bottleneck beyond Earth, and (c) closed interplanetary magnetic loop, injecting particles along the near leg of the loop that undergo reflection in the far leg, or injecting particles along both legs of the loop. Configurations (b) and (c) could be caused by a previous coronal mass ejection. The only case that provides a good fit to the data is injection along both legs of a closed interplanetary magnetic loop.

Figure 2. (right side) Fits (solid lines) to density (points, left panels) and weighted anisotropy (points, right panels) as a function of time for an optimized injection function near the Sun, for (a-b) a spiral magnetic field, (c-d) a magnetic bottleneck beyond Earth, (e-f) injection along the near leg of a closed interplanetary magnetic loop, and (g-h) equal injection along both legs of a closed interplanetary magnetic loop, the only case that provides a good fit to the data.

2. Observed time profiles can be understood in terms of standard transport processes of scattering and focusing, with the additional process of escape from the loop.

3. Relativistic solar protons were injected near the Sun starting at 17:46 UT and peaking at 17:51 UT, with a FWHM duration of 6 minutes (all \pm 2 minutes). There was also extended injection for $\approx 90$ minutes 
at a lower level, followed by a cutoff presumably associated with changing magnetic connection to the source.

4. Observed pitch angle distributions indicate $q<1$, where $q$ is a transport parameter identified with the spectral index of magnetic turbulence. To our knowledge such a low value has not previously been inferred from solar particle observations.

5. Relativistic protons escaped from the loop with a time constant of 180 minutes, which is interpreted as an escape time due to transport perpendicular to the large-scale magnetic field.

6. The length of the loop is inferred to be $4.7 \pm 0.3 \mathrm{AU}$, and the parallel mean free path is estimated as 1.2 to $2.0 \mathrm{AU}$, depending on the value of $q$.

7. These results are consistent with an overall picture from various reports in the literature of low magnetic fluctuations, very low slab fluctuations, and long scattering mean free paths in magnetic loops and other regions of high Alfvén speed. The present results suggest that the turbulent correlation length can be unusually short in magnetic loops.

\section{Acknowledgements}

We thank our colleagues at IZMIRAN (Russia), the Polar Geophysical Institute (Russia), and the Australian Antarctic Division for furnishing data. We also thank Pete Riley, Dermott Mullan, and Alejandro Sáiz for useful discussions. Terre Adelie data were kindly provided by the French Polar Institute (IPEV, Brest) and by Paris Observatory. This research was partially supported by the Thailand Research Fund, the Rachadapisek Sompoj Fund of Chulalongkorn University, and the US National Science Foundation (grant ATM-0000315).

\section{References}

[1] J. W. Bieber, P. Evenson, and M. A. Pomerantz, Eos Trans. AGU, 71, 1027 (1990).

[2] J. A. Earl, Astrophys. J., 206, 301 (1976).

[3] M. A. Shea and D. F. Smart, 25th ICRC, Durban (1997) 1, 129.

[4] J. L. Cramp, M. L. Duldig, E. O. Flückiger, J. E. Humble, M. A. Shea, and D. F. Smart, J. Geophys. Res., 102, 24237 (1997).

[5] E. O. Flückiger, E. Kobel, D. F. Smart, M. A. Shea, J. E. Humble, J. L. Cramp, and M. L. Duldig, Eos Trans. AGU, 74(43), Fall Meet. Suppl., 487 (1993).

[6] J. W. Bieber et al., Astrophys. J., 567, 622 (2002).

[7] D. Ruffolo, Astrophys. J., 382, 688 (1991).

[8] T. Nutaro, S. Riyavong, and D. Ruffolo, Comp. Phys. Comm., 134, 209 (2001).

[9] D. Ruffolo, T. Khumlumlert, and W. Youngdee, J. Geophys. Res., 103, 20591 (1998). 